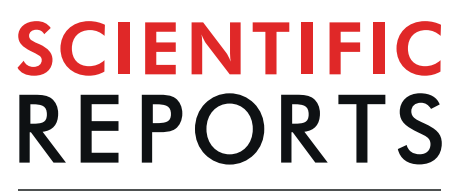

natureresearch

\title{
Degeneracy in the emergence of spike-triggered average of hippocampal pyramidal neurons
}

\begin{abstract}
Abha Jain ${ }^{1,2}$ \& Rishikesh Narayanan ${ }^{1 *}$
Hippocampal pyramidal neurons are endowed with signature excitability characteristics, exhibit theta-frequency selectivity - manifesting as impedance resonance and as a band-pass structure in the spike-triggered average (STA) - and coincidence detection tuned for gamma-frequency inputs. Are there specific constraints on molecular-scale (ion channel) properties in the concomitant emergence of cellular-scale encoding (feature detection and selectivity) and excitability characteristics? Here, we employed a biophysically-constrained unbiased stochastic search strategy involving thousands of conductance-based models, spanning 11 active ion channels, to assess the concomitant emergence of 14 different electrophysiological measurements. Despite the strong biophysical and physiological constraints, we found models that were similar in terms of their spectral selectivity, operating mode along the integrator-coincidence detection continuum and intrinsic excitability characteristics. The parametric combinations that resulted in these functionally similar models were non-unique with weak pair-wise correlations. Employing virtual knockout of individual ion channels in these functionally similar models, we found a many-to-many relationship between channels and physiological characteristics to mediate this degeneracy, and predicted a dominant role for HCN and transient potassium channels in regulating hippocampal neuronal STA. Our analyses reveals the expression of degeneracy, that results from synergistic interactions among disparate channel components, in the concomitant emergence of neuronal excitability and encoding characteristics.
\end{abstract}

Degeneracy, the ability of distinct structural components to elicit similar physiology, is ubiquitous in biology, across organisms and across scales ${ }^{1-8}$. Several studies have shown that models with disparate combinations of ion channel conductances can have physiological measurements with values within their experimentally determined ranges $^{4,6,8-19}$. Most of these studies, however, have focused largely on measures of excitability, with the focus primarily on homeostasis. However, neurons are also endowed with specific physiological properties that define the features that they encode, their operating mode (i.e., their position along the integrator-coincidence detector (I-CD) continuum), and coincidence detection capabilities ${ }^{20-26}$. Could degeneracy be a substrate for neurons to encode specific characteristics, to be endowed with specific operational characteristics and still maintain their excitability properties within experimental bounds? Could the feature detection/extraction goals of a neuron coexist with its ability to maintain specific levels of excitability? Could disparate combinations of ion channels mediate such co-existence? Is there a dominance hierarchy among different channels in determining a neuron's operating mode?

To address these questions, we carried out computational analyses that employed hippocampal CA1 pyramidal neuron models as substrates. We used this particular category of neurons, on account of electrophysiological data availability on ion channel kinetics ${ }^{27-33}$, intrinsic excitability measurements ${ }^{28,29,34-37}$ and measurements of their encoding and operational characteristics ${ }^{25}$. The encoding and operational characteristics of these models were quantified using measurements dependent on the spike-triggered average (STA). The STA of a neuron, defined as the average current stimulus that is required to elicit a spike, is a powerful physiological measurement that represents both encoding and excitability properties of a neuron. The STA provides quantitative assessments of intrinsic excitability, spectral selectivity and neuronal operating mode along the integrator-coincidence detector (I-CD) continuum. These quantifications, showing the STA of hippocampal pyramidal neurons to be endowed with theta-frequency $(4-10 \mathrm{~Hz})$ selectivity and gamma-range $(25-150 \mathrm{~Hz})$ coincidence detection capabilities $^{23-26}$, place tight constraints on channel properties that result in their emergence ${ }^{20-26}$. 
In addition to STA measurements, in our analyses, we placed several available electrophysiological constraints on intrinsic excitability characteristics ${ }^{35,36,38,39}$. Specifically, we employed excitability measures based on pulse current injections (e.g., input resistance, firing rate) along with measurements that employ input frequency as a variable (e.g., impedance amplitude and phase, resonance frequency). These measurements provided us with the opportunity to physiologically constrain model populations, while also enabling us to assess heterogeneity and degeneracy in these populations. The availability of both encoding as well as excitability measurements enabled us to constrain the operating mode of a given neuron model in a physiologically realistic setting (i.e. in the central region, rather than at the more abstract ends of the I-CD continuum ${ }^{20}$ ) as well as study the concomitant constraints on neuronal excitability. In addition to these physiological constraints on measurements at the cellular scale (neuronal encoding and excitability), we placed explicit biophysical constraints on our models at the molecular scale (ion channels and their properties) based on electrophysiological recordings. We demonstrate, using an unbiased stochastic search algorithm involving thousands of models that yielded biophysically constrained and physiologically validated models, that neurons with disparate channel conductances can concomitantly possess similar encoding and excitability characteristics.

Additionally, we determined the manner in which different ion channels influence various parameters of a neuron's STA by generating acute virtual knockouts ${ }^{9,14,40}$ of individual ion channels, and by assessing the STA of each virtual knockout model (VKM). We demonstrate that both frequency selectivity and coincidence detection measured from neuronal STA are critically reliant on several ion channels, implying that strong interactions among different ion channels drive operational characteristics and excitability homeostasis in hippocampal pyramidal neurons. These analyses also showed that $\mathrm{HCN}$ and transient $\mathrm{K}^{+}$channels are the predominant intrinsic regulators of the hippocampal pyramidal neuron's operating mode. Our results provide experimentally well-constrained lines of evidence for the expression of degeneracy in the ability of single neurons to be endowed with similar encoding and excitability characteristics, and in determining their operational mode along the I-CD continuum.

\section{Results}

Base model and its measurements. A cylindrical base model was created with both length $(L)$ and diameter $(d)$ kept at $105 \mu \mathrm{m}$ (Fig. 1 ); $R_{\mathrm{m}}$ and $C_{\mathrm{m}}$ were kept at $40 \mathrm{k} \Omega . \mathrm{cm}^{2}$ and $1 \mu \mathrm{F} / \mathrm{cm}^{2}$ respectively, to set the passive input resistance $\left(R_{\mathrm{m}} / \pi d L\right)$ to be around $120 \mathrm{M} \Omega^{35}$ and the passive time constant $\left(R_{\mathrm{m}} C_{\mathrm{m}}\right)$ to be $40 \mathrm{~ms}^{36}$. We introduced the following ion channels, with kinetics and gating properties derived from hippocampal pyramidal neurons, into this passive structure: fast sodium $(\mathrm{NaF})$, delayed rectifier potassium (KDR), $A$-type potassium (KA), $M$-type potassium (KM), hyperpolarization-activated cyclic-nuclotide gated (HCN) nonspecific cation, $L$-type calcium $(\mathrm{CaL}), N$-type calcium $(\mathrm{CaN}), R$-type calcium $(\mathrm{CaR}), T$-type calcium $(\mathrm{CaT})$, big- $(\mathrm{BK})$ and small-conductance (SK) calcium-activated potassium channels. Conductances of the different ion channels were hand-tuned such that values for all 12 intrinsic measurements (Fig. 1b-j) fell in their appropriate experimentally determined ranges (conductance values are shown in Table 1). These base model parametric values then provided the span for the stochastic search that was performed on the different parametric combinations (Table 2). Apart from the match in excitability measurements with their respective experimental counterparts ${ }^{15,35,36,38}$, we noted that the STA associated with the base model (Fig. 1 j) was endowed with a small negative lobe, yielding an $f_{\text {STA }}$ (Fig. 1k) within the valid theta range of frequencies ${ }^{25}$. The base model measurements also showed that the effective coincidence detection window (ECDW) was in the gamma frequency range (Fig. 1j), another characteristic feature of the STA associated with CA1 pyramidal neuronal soma ${ }^{25}$.

Stochastic sampling and validation. We stochastically generated 5000 unique models by sampling uniform distributions for 14 parameters (all ion channel conductances, the specific membrane capacitance, and the decay time constant of calcium), with each distribution centered about the corresponding value in the base model (Table 1). Validity of each model was then ascertained by checking if all the 12 intrinsic measurements computed from these models fell within their respective electrophysiological ranges (Table 2). Of the 12 measurements, five were derived from the STA that characterized the operating mode and coincidence detection windows. The other measurements defined intrinsic excitability and frequency-dependent neuronal response properties in the subthreshold range. Of the 5000 models generated, we found 72 models ( $1.5 \%$ of the total number of models) that satisfied all these validity criteria and were declared as valid models.

Ion channel degeneracy in the co-emergence of STA characteristics and intrinsic excitability measurements. To address our primary question on whether degeneracy could be a substrate for neurons to both possess specific operational characteristics and maintain their intrinsic excitability properties, we assessed the parametric ranges of these 72 valid models. As a direct consequence of the validation criteria employed in our analyses, these 72 models satisfied all encoding and excitability constraints that we had placed. Were the 14 model parameters (Table 1) that defined these 72 models organized into clusters or were they distributed? A distributed set of underlying parameters, where different sets of channel conductances yield similar function, would constitute a direct line of evidence for the expression of degeneracy in this model population.

To assess this, we chose five models whose intrinsic measurements (both excitability measurements and STA measurements) were very similar (Fig. $2 \mathrm{a}-\mathrm{i}$ ). We then plotted the value of each of the 14 parameters (normalized individually with reference to their respective min and max values in Table 1) for all these five models (Fig. 2j) to assess if they were clustered or were disparate across distinct models. We noted that most parametric values spanned their entire respective ranges, suggesting the absence of clustering or the need to maintain individual conductances at specific values for achieving functional similarity. This provided us with the first line of evidence that degeneracy could indeed provide a substrate for neurons to concomitantly maintain their encoding/ 
a

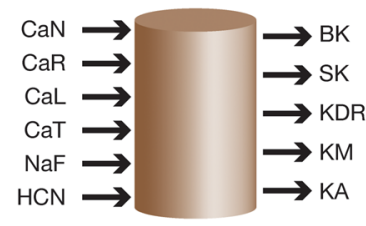

e

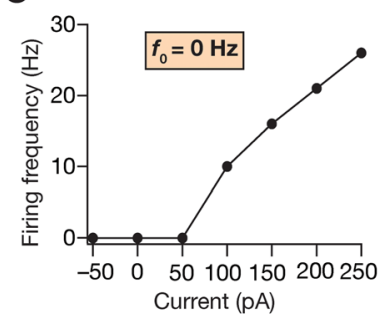

i

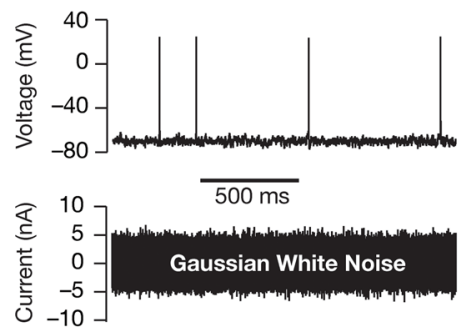

b

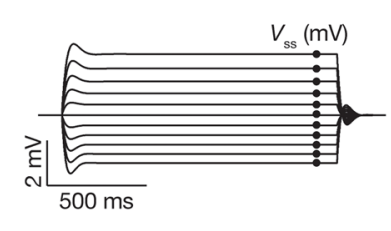

C

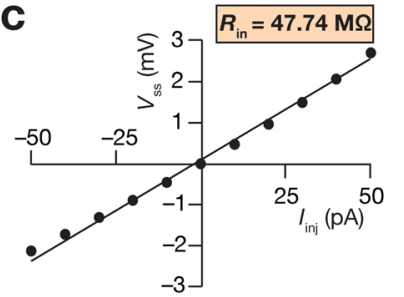

d

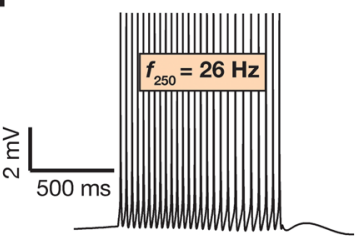

$\mathbf{f}$

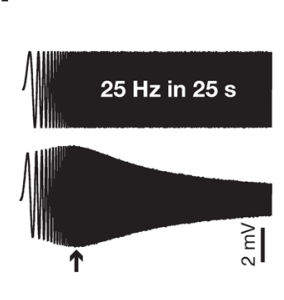

g

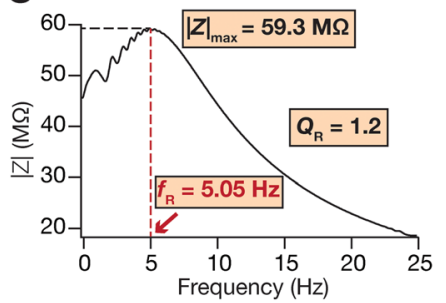

h

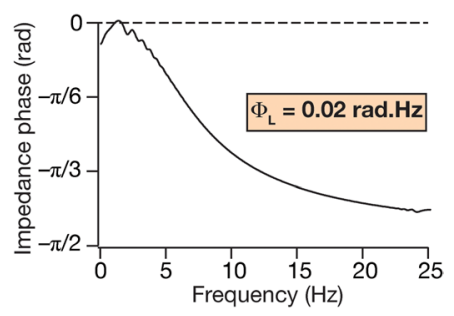

$\mathbf{k}$

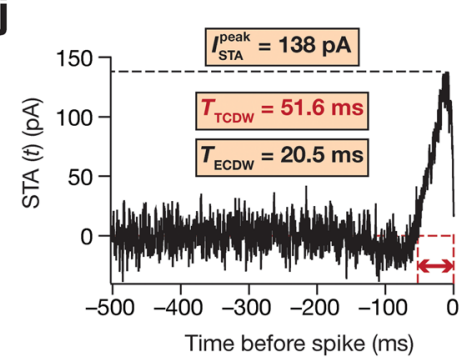

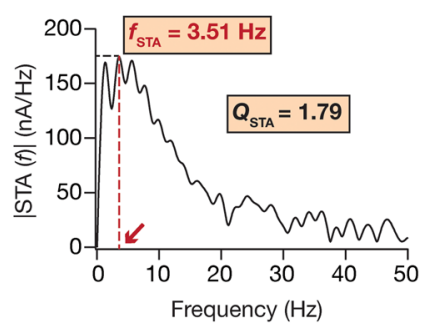

Figure 1. Illustration of physiological measurements employed in this study using the base model. (a) Depiction of the cylindrical single compartment base model, along with the ion channels that were inserted in it. Arrows pointing towards the cylinder represent inward currents and arrows pointing away from the cylinder represent outward currents. (b) Voltage traces in response to a $1000 \mathrm{~ms}$ current injection, from $-50 \mathrm{pA}$ to $+50 \mathrm{pA}$, in steps of $10 \mathrm{pA} . V_{\mathrm{ss}}$ represents the steady state voltage (black circles) achieved with each current injection. (c) Plot of the steady-state voltage obtained from the traces in panel $\mathbf{b}$ versus the corresponding current amplitude. Slope of the linear fit to this $V-I$ curve defines input resistance $\left(R_{\mathrm{in}}\right)$. (d) Voltage trace in response to a $250 \mathrm{pA}$ current injection, lasting for $1000 \mathrm{~ms}$. The number of action potentials fired during this period is measured as $f_{250}$. (e) $f-I$ curve for the base model, plotting firing frequency for different amplitudes of current injection. $f_{0}$ measured the firing frequency when no current was injected. (f) Upper panel represents the chirp current stimulus of 20 pA amplitude (peak-to-peak), and with a frequency that increases linearly from $0 \mathrm{~Hz}$ to $25 \mathrm{~Hz}$ in $25 \mathrm{~s}$. Lower panel represents the voltage response of the neuron to such a chirp stimulus. The black arrow depicts the location of maximal voltage response. (g) Impedance amplitude profile obtained from the voltage response to the chirp stimulus, both shown in panel f. $|Z|_{\max }$ represents the maximal impedance amplitude; resonance frequency $f_{\mathrm{R}}$ is the frequency at which $|Z|_{\max }$ is achieved (red arrow) and $Q_{\mathrm{R}}$ is the resonance strength. (h) Impedance phase profile obtained from the voltage response to the chirp stimulus, both shown in panel $\mathbf{f}$. Total inductive area $\Phi_{\mathrm{L}}$ is area under the positive part of this impedance phase profile. (i) Lower panel represents one part of the Gaussian white noise (GWN) current stimulus. Upper panel represents the voltage response of the neuron to this GWN current stimulus. (j) STA trace for the base model. On the $X$-axis is the time before generation of an action potential in ms, while the $Y$-axis is STA current in pA. $I_{S T A}^{\text {peak }}$ represents the STA peak current; $T_{\mathrm{TCDW}}$ (window depicted by the red arrows) and $T_{\mathrm{ECDW}}$ represent the total and the effective coincidence detection windows, respectively. (k) Fourier transform of the STA from panel $\mathbf{j}$ that reveals its spectral selectivity at the STA characteristic frequency $\left(f_{\mathrm{STA}}\right.$; red arrow). $Q_{\mathrm{STA}}$ is the selectivity strength.

operational characteristics along with their excitability characteristics, whereby disparate sets of channels could result in the joint emergence of both physiological signatures.

The choice of five hand picked similar models where there was evidence for degeneracy presents a demonstration of the expression of degeneracy with reference to these physiological measurements. Do these conclusions extend to all the valid models in the populations? To address this, we plotted the values for the 10 intrinsic measurements (excluding $f_{0}$ and $f_{250}$, for all the 72 valid models), and by virtue of the validation process, they fell within the appropriate experimentally determined ranges (Fig. 3), while also confirming the presence of heterogeneity in these models. However, when we plotted the histograms of individual parameters for all 72 models, we found the histograms to span the entire min-max range (Table 2) of the respective parameter (Fig. 4a, bottom most panels).

Although the widespread ranges of individual parameters strongly point to disparate parametric combinations yielding similar function, could this be because different ion channels have pairwise relationships in their conductance values? To address this, we computed pair-wise correlations across all 14 parameters for each of the 


\begin{tabular}{|l|l|l|l|l|}
\hline & Neuronal Parameter & Symbol & $\begin{array}{l}\text { Value in } \\
\text { base-model }\end{array}$ & Testing range \\
\hline 1 & Specific membrane capacitance, $\mu \mathrm{F} / \mathrm{cm}^{2}$ & $C_{\mathrm{m}}$ & 1.00 & 0.75 to 1.50 \\
\hline 2 & Specific membrane resistance $\left(R_{\mathrm{m}}\right), \mathrm{k} \Omega \cdot \mathrm{cm}^{2}$ & $R_{\mathrm{m}}$ & 40 & 20 to 80 \\
\hline 3 & Decay time constant of calcium dynamics, $\mathrm{ms}$ & $\tau_{\mathrm{Ca}}$ & 33 & 10 to 300 \\
\hline & Maximal conductance of: & & & \\
\hline 4 & NaF channel, $\mathrm{mS} / \mathrm{cm}^{2}$ & $g_{\mathrm{NaF}}$ & 14.0 & 7.0 to 28.0 \\
\hline 5 & HCN channel, $\mu \mathrm{S} / \mathrm{cm}^{2}$ & $g_{\mathrm{HCN}}$ & 31.8 & 15.9 to 63.6 \\
\hline 6 & KDR channel, $\mathrm{mS} / \mathrm{cm}^{2}$ & $g_{\mathrm{KDR}}$ & 6.05 & 3.025 to 12.10 \\
\hline 7 & CaT channel, $\mathrm{mS} / \mathrm{cm}^{2}$ & $g_{\mathrm{CaT}}$ & 1.55 & 0.75 to 3.0 \\
\hline 8 & CaL channel, $\mathrm{mS} / \mathrm{cm}^{2}$ & $g_{\mathrm{CaL}}$ & 0.5 & 0.25 to 1.0 \\
\hline 9 & CaR channel, $\mathrm{mS} / \mathrm{cm}^{2}$ & $g_{\mathrm{CaR}}$ & 0.1 & 0.05 to 0.2 \\
\hline 10 & CaN channel, $\mathrm{mS} / \mathrm{cm}^{2}$ & $g_{\mathrm{CaN}}$ & 0.1 & 0.05 to 0.2 \\
\hline 11 & KA channel, $\mathrm{mS} / \mathrm{cm}^{2}$ & $g_{\mathrm{KA}}$ & 6.06 & 3.03 to 12.12 \\
\hline 12 & KM channel, $\mu \mathrm{S} / \mathrm{cm}^{2}$ & $g_{\mathrm{KM}}$ & 0.1 & 0.05 to 0.2 \\
\hline 13 & SK channel, $\mu \mathrm{S} / \mathrm{cm}^{2}$ & $g_{\mathrm{SK}}$ & 0.01 & 0.005 to 0.02 \\
\hline 14 & BK channel, $\mu \mathrm{S} / \mathrm{cm}^{2}$ & $g_{\mathrm{BK}}$ & 0.5 & 0.25 to 1.0 \\
\hline
\end{tabular}

Table 1. List of parameters and ranges of the uniform distributions used.

\begin{tabular}{|l|l|l|l|}
\hline & Intrinsic Measurement & $\begin{array}{l}\text { Lower } \\
\text { bound }\end{array}$ & $\begin{array}{l}\text { Upper } \\
\text { bound }\end{array}$ \\
\hline 1 & Input resistance (in $\mathrm{M} \Omega$ ) & 30 & 90 \\
\hline 2 & Firing frequency for 0 pA current injection (in $\mathrm{Hz})$ & 0 & 0 \\
\hline 3 & Firing frequency for 250 pA current injection (in $\mathrm{Hz})$ & 20 & 35 \\
\hline 4 & Resonance frequency (in Hz) & 2.0 & 5.5 \\
\hline 5 & Resonance strength & 1.05 & 1.5 \\
\hline 6 & Maximum impedance amplitude (in $\mathrm{M} \Omega$ ) & 50 & 110 \\
\hline 7 & Total inductive phase (in radian.Hz) & 0 & 0.20 \\
\hline 8 & STA characteristic frequency (in Hz) & 3.0 & 7.0 \\
\hline 9 & STA selectivity strength & 1.5 & 2.2 \\
\hline 10 & Peak STA current (in pA) & 90 & 900 \\
\hline 11 & Total coincidence detection window (in $\mathrm{ms})$ & 25 & 62 \\
\hline 12 & Effective coincidence detection window (in $\mathrm{ms})$ & 5 & 25 \\
\hline
\end{tabular}

Table 2. Bounds on intrinsic measurements used to define valid model population. Electrophysiological bounds for measurements 1-7 were obtained from (Narayanan and Johnston, 2007, 2008; Narayanan et al., 2010 and Malik et al., 2016) ${ }^{35,36,38,39}$ and STA measurements 8-12 were obtained from (Das and Narayanan, 2017) ${ }^{25}$.

72 models (Fig. 4a-b). We found that $97 \%$ ( 88 out of 91 ) of the correlations were weak or very weak ${ }^{41}$, with values between -0.4 and 0.4 (Fig. $4 \mathrm{~b}$ ). These observations showed the absence of strong cross-dependencies across channel conductances and other parameters in the concomitant emergence of STA-based and excitability measurements. Together, these results demonstrate that ion channel degeneracy could be a substrate for neurons to possess specific operational characteristics and still maintain their intrinsic excitability properties.

Finally, we asked if there were significant cross-dependencies in the distinct measurements we had employed in our validation process. This is important because if the measurements had significant correlations between them, it would imply that we were not significantly constraining the parameters to suppress the potential expression of degeneracy. These correlations also allow us to arrive at another layer of physiological validation by comparing them with their electrophysiological counterparts ${ }^{25}$. To assess this, we determined the pairwise correlations amongst 10 intrinsic measurements for all the 72 valid models (Fig. 5). Consistent with previous experimental observations from CA1 pyramidal neurons ${ }^{25,42}$, we observed (Fig. 5) weak correlation coefficients for a large proportion of these pairwise comparisons ( 38 of 45 , or $84 \%$, with values between -0.4 to $0.4^{41}$ ), suggesting that there were no strong cross-dependencies across these measurements. However, in a manner consistent with prior experimental observations ${ }^{25,42}$, we observed strong positive correlations between excitability measurements $\left(R_{\mathrm{in}}\right.$ and $\left.|Z|_{\mathrm{max}}\right)$ and coincidence detection metrics $\left(T_{\mathrm{TCDW}}\right.$ and $\left.T_{\mathrm{ECDW}}\right)$. These observations demonstrate that the different measurement constraints on the model were not correlated, thereby ensuring that the model was subjected to strong constraints that reflected different functional characteristics.

If these measurements indeed reflected different functional characteristics, validity or invalidity of a specific model to one of these measurements should not be predictive of validity or invalidity of the same model to another measurement. To test if this was indeed the case, we first took the six standard intrinsic measurements $\left(R_{\mathrm{in}}, f_{250},|Z|_{\text {max }}, f_{\mathrm{R}}, Q_{\mathrm{R}}, \Phi_{\mathrm{L}}\right)$ of all the 5000 models. For any given pair of measurements (say, $\mathrm{A}$ and $\mathrm{B}$ ) 
a

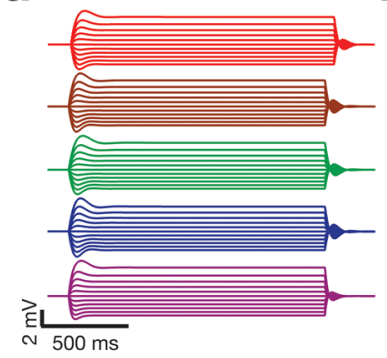

b

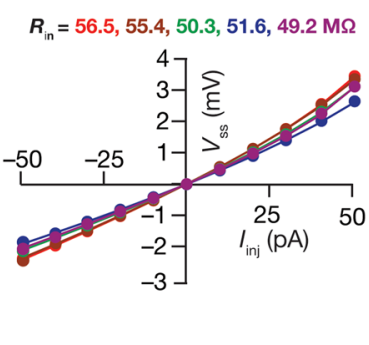

f

e

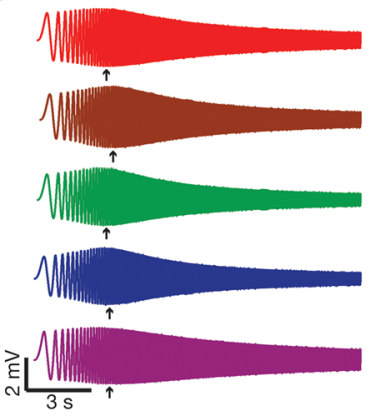

i

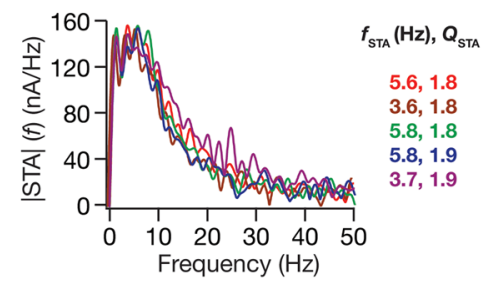

j
C

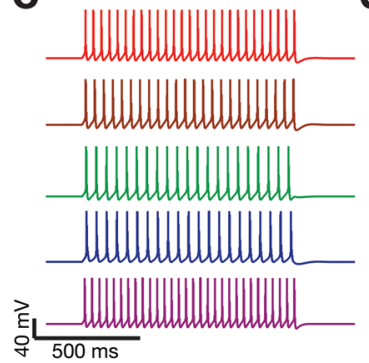

$\mathrm{g}$ d

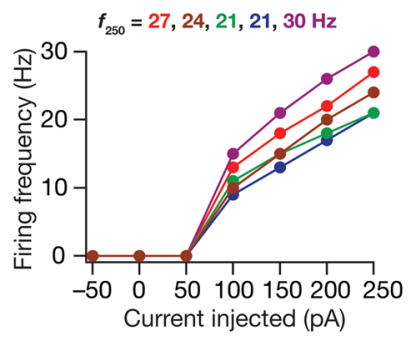

h

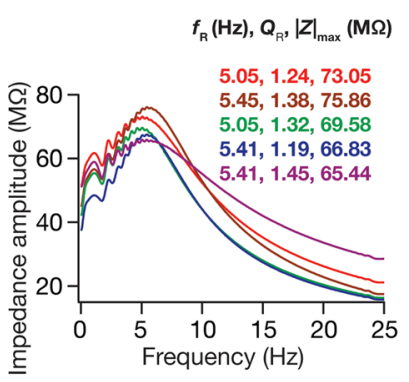

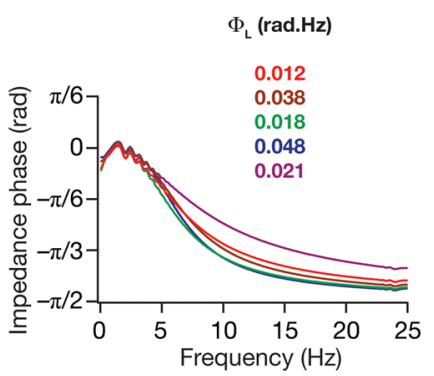

$i_{\mathrm{STA}}^{\text {peak }}(\mathrm{pA}), T_{\mathrm{TCDW}}(\mathrm{ms}), T_{\mathrm{ECDW}}(\mathrm{ms})$

$115,52.6,21.0$ $117,57.3,22.8$ $119,56.6,22.8$ $117,60.5,24.5$

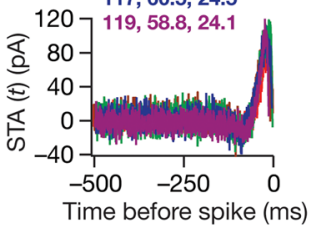

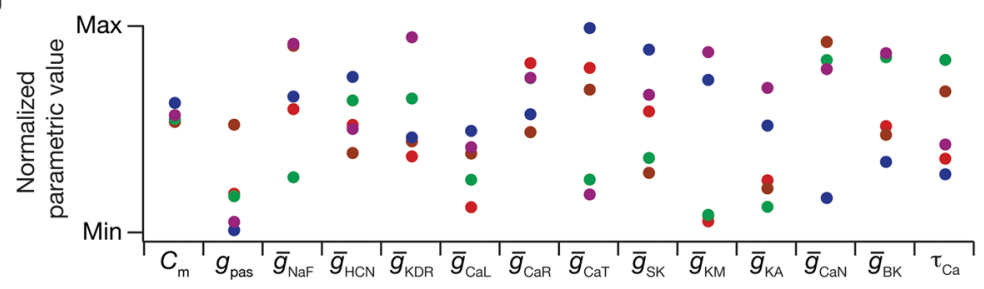

Figure 2. Disparate molecular-scale parameters yielded models with similar cellular-scale physiological measurements, spanning encoding and excitability characteristics. The voltage traces and parameters correspond to 5 models that satisfied all the validity criteria presented in Table 2. The color codes for model characteristics are maintained across panels. (a) Voltage responses of the 5 models to current injections spanning the range $-50 \mathrm{pA}$ to $+50 \mathrm{pA}$ in increments of $10 \mathrm{pA}$. (b) Plot of the steady-state voltage responses obtained from panel a versus the corresponding current injection to obtain $V$ - $I$ curves and input resistance $\left(R_{\text {in }}\right)$. (c) Voltage responses of the 5 models to a $250 \mathrm{pA}$ current injection. (d) The $f-I$ curves for the 5 models. (e) Voltage traces obtained from the five models in response to the chirp stimulus. Arrows represent the locations of the maximal voltage response. (f) Impedance amplitude profiles for the five models, derived from the chirp stimulus responses shown in panel e. (g) Impedance phase profile for the five models, derived from the chirp stimulus responses shown in panel c. (h-i) STA traces (h) and their Fourier spectra (i) for the five models. (j) Distribution of the 14 parameters that governed the 5 model neurons, normalized with reference to their respective min-max ranges (Table 1).

from these 5000 models, we constructed three matrices assessing pair-wise dependencies of validity across these measurements: (i) the valid-valid matrix showed the number of models that were also valid for measurement A when they were valid for measurement B (Supplementary Fig. S1a); (ii) the invalid-invalid matrix depicted the number of models that were also invalid for measurement A when they were invalid for measurement $B$ (Supplementary Fig. S1b); and (iii) the valid-invalid matrix provided number of models that were valid for measurement A, but were invalid for measurement B (Supplementary Fig. S1c). If these measurements were redundant in terms of their selection pressure on the model validation process, then validation/invalidation for one measurement should predict the validation/invalidation of the other redundant measurement. However, in our case, we found from these matrices that validity (or invalidity) for one measurement did not predict validity (or invalidity) for any of the other intrinsic measurements.

We repeated these analyses with the 5 STA measurements for the 355 (of the total 5000) models that satisfied all the intrinsically physiological criteria (Supplementary Fig. S1d-f), and arrived at similar conclusions for STA measurements as well. In addition, these analyses also demonstrated that there were models that were valid for all excitability measurements, but did not satisfy validity constraint for one or the other STA measurement (Supplementary Fig. S1d,f). Together, our analyses demonstrated that the set of measurements employed in this study were not redundant in terms of their selection pressure on the model validation process. 

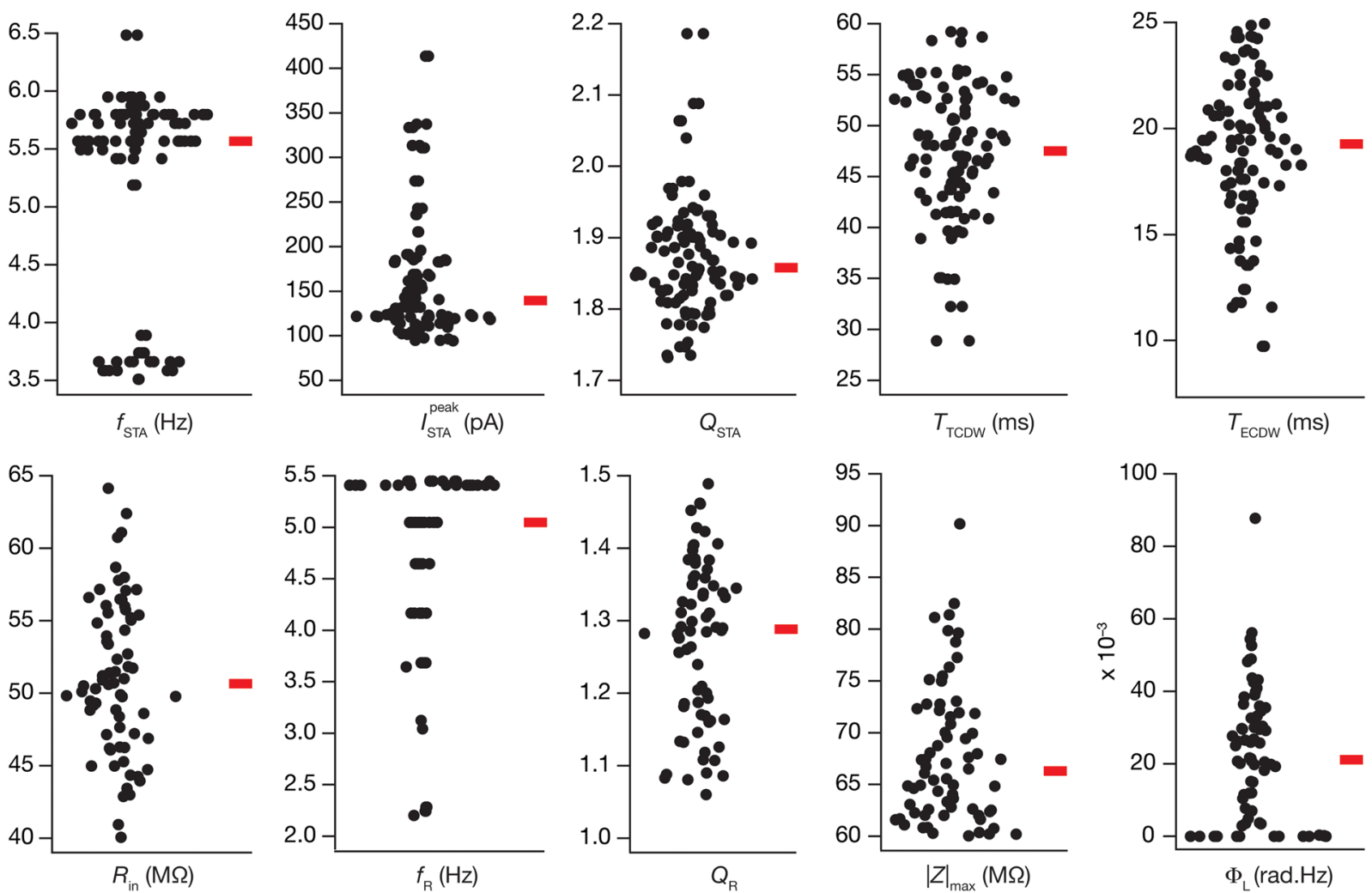

Figure 3. Distribution of neuronal intrinsic physiological characteristics for the valid model population. Each sub-panel depicts the population distribution of a given intrinsic measurement for the 72 valid models, shown with the median value, indicated by the red bar. Compare ranges with validity criteria specified in Table 2 . The theta-frequency range of $f_{\mathrm{STA}}$ and gamma-frequency range of $T_{\mathrm{ECDW}}$ may be noted for all models.

Virtual knockout models unveiled a many-to-many relationship between channels and STA measurements. Virtual knockout models (VKM) are an ideal way to assess the impact of individual channels on physiological measurements within the degeneracy framework that involve heterogeneous populations $s^{9,14,40,43}$. For each valid model, we generated virtual knockouts (by setting the respective conductance to zero, without altering any other parameter) with reference to 9 ion channels (the spike-generating conductances were not knocked out, given that they are essential for STA computations): CaT, CaR, CaN, CaL, KM, KA, SK, BK and HCN (Supplementary Fig. S2). We compared the 5 STA-based measurements of every VKM (across 9 channels) with the corresponding baseline values (Supplementary Figs. S3-S5). For all VKMs (corresponding to each of the 9 channels), we found all STA measurements to be significantly different (Wilcoxon rank sum test, $p<0.05)$ compared to the corresponding values in their respective base models (Supplementary Figs. S3-S5). From these analyses, we noted the following (Fig. 6, Supplementary Figs. S2-S5): (i) the impact of knocking out different channels on specific measurements was differential, whereby different channels had distinct effects on these measurements; (ii) for a given ion channel and a given measurement, there was significant variability in the impact of the knockout, even involving the direction of change in some cases; and (iii) knocking out a specific channel altered several STA measurements and any given measurement was altered with knockout of several channels, implying a many-to-many relationship between channel conductances and STA measurements.

To assess the specifics of the impact of channels on STA measurements, we plotted the distribution of the knockout-induced changes in each STA measurement for each of the 9 different channels (Fig. 6). Although there was significant heterogeneity in the impact of different channels, we noted that the HCN and KA VKMs resulted in a reduction, relative to their respective pre-knockout values, of $>60 \%$ in the $f_{\text {STA }}$ across models (Fig. 6a). We noted this to be consistent with previous computational and electrophysiological studies on hippocampal pyramidal neurons, which have demonstrated that blocking $\mathrm{HCN}$ channels reduces its $f_{\mathrm{STA}}{ }^{23-25}$. We also noted that no particular ion channel caused either a consistent reduction or augmentation of the $Q_{\text {STA }}$ (Fig. 6b).

We observed that the peak STA current $\left(I_{S T A}^{\text {peak }}\right)$ was consistently lower in the case of the HCN and KA VKMs, with VKMs of other channels showing considerable variability with reference to their impact on $I_{S T A}^{\text {peak }}$ (Fig. 6c). As $I_{S T A}^{\text {peak }}$ is a measure of excitability with lower $I_{S T A}^{\text {peak }}$ (i.e. the average current required for triggering an action potential is lower) translating to higher excitability, these observations imply that the presence and activation of either channel reduces the neuron's excitability ${ }^{23-25}$. However, around half of the CaT VKMs showed an increase in the peak STA current compared to their baseline values, with the other half manifesting a decrease after knockout of CaT channels. We noted that this could be an effect of the expression of SK channels in our model. In models where the SK channel density was larger, the knockout of CaT channels would lead to a significant reduction of SK current, thereby leading to an increase in excitability, whereas in models where the SK channel density was 
a

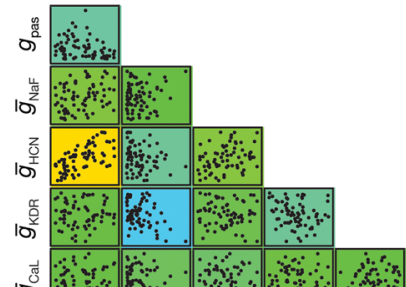

b

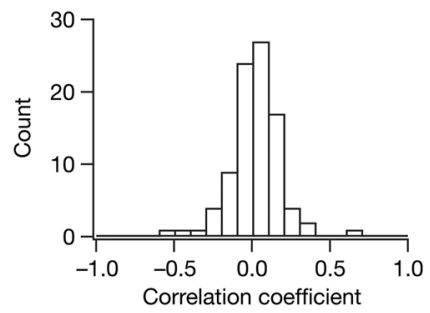

$100^{\circ}$

100

$10)^{\frac{x}{5}}$

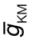

$1 0 \longdiv { 5 }$

10)

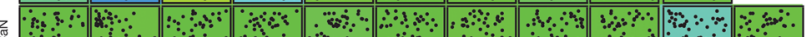

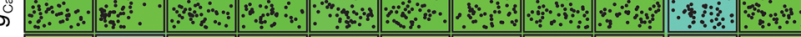

o
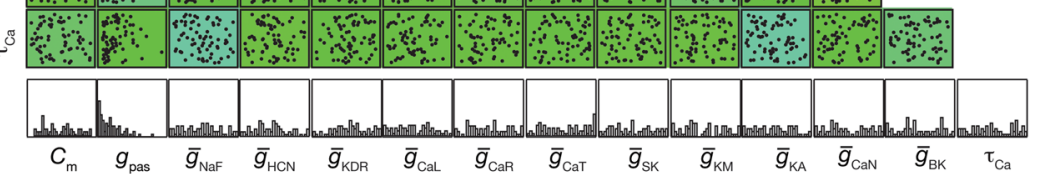

Figure 4. Broad distributions of and weak pairwise correlations amongst parameters of the valid model population unveiled parametric degeneracy in the concomitant expression of excitability and encoding measurements. (a) Lower diagonal of a matrix depicting pairwise relationships between the 14 parametric values of the 72 valid models. Each subplot is a scatter plot depicting values of the two parameters, labeled to the bottom and the left. The lowermost row comprises normalized histograms of the 14 parameters across all 72 valid models, and may be noted to have a broad distribution spanning the entire parametric range. These plots are overlaid on the lower diagonal of a color-coded matrix constructed from the respective Pearson correlation coefficient values. (b) Histogram of the correlation coefficients obtained from the scatter plots in panel a.

a

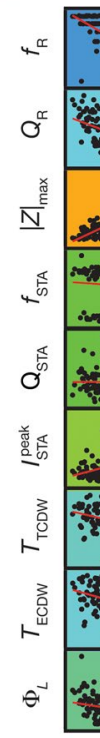

b

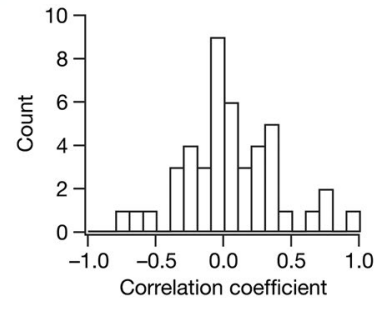

Figure 5. Intrinsic measurements of the valid-model population exhibited differential correlations. (a) Pairwise scatter plots with respective linear fits (red lines) across the 10 intrinsic measurements for the 72 valid models. These plots are overlaid on the lower diagonal of a color-coded matrix, containing the respective correlation coefficient values. (b) Histogram of the correlation coefficients obtained from the scatter plots in panel a. 


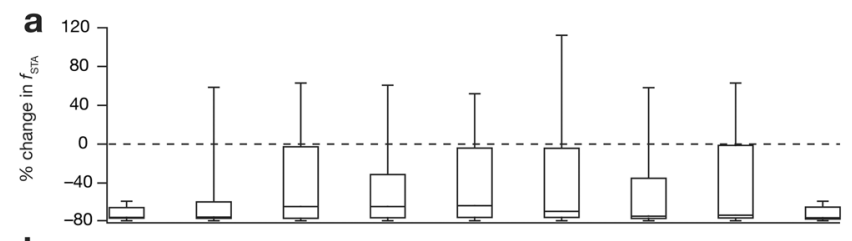

b
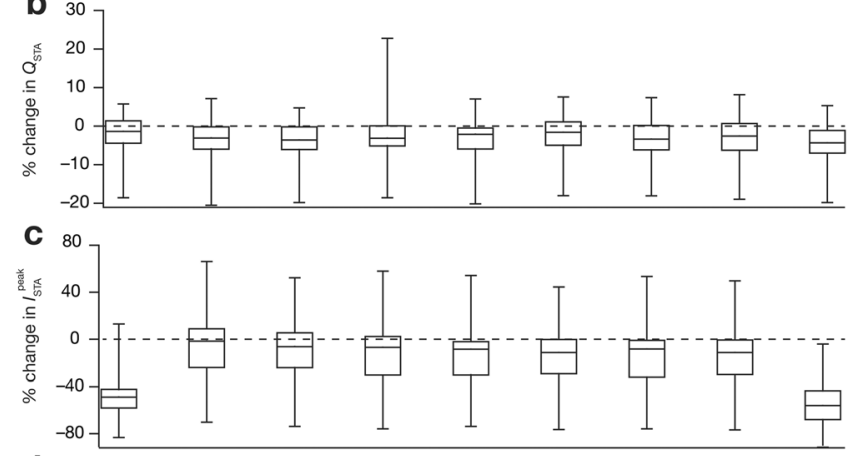

d

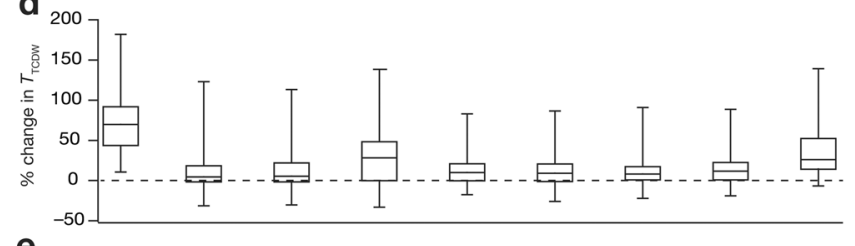

$$
\begin{aligned}
& \text { e }
\end{aligned}
$$

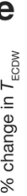$$
\begin{aligned}
& 300 \\
& 200 \\
& 0
\end{aligned}
$$

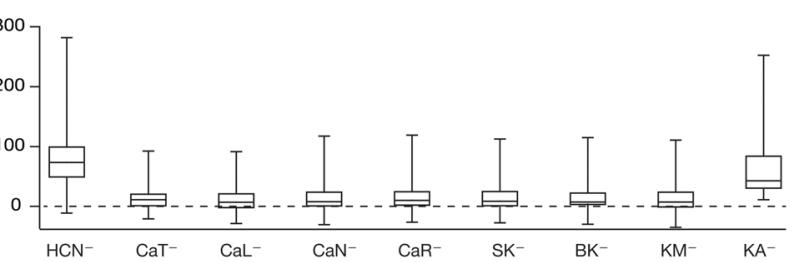

Figure 6. Virtual knockout simulations unveiled a many-to-many relationship between channel subtypes and intrinsic measurements. Distributions of percentage changes (represented as quartiles) for STA characteristics frequency $f_{\text {STA }}(\mathbf{a})$, STA selectivity strength $Q_{\text {STA }}(\mathbf{b})$, peak STA current $I_{\text {STA }}^{\text {peak }}(\mathbf{c})$, total coincidence detection window $T_{\mathrm{TCDW}}(\mathbf{d})$ and effective coincidence detection window $T_{\mathrm{ECDW}}(\mathbf{e})$. Each column corresponds to a specific channel knockout, as indicated by the label at the base of the figure. Also see Supplementary Figs. S2-S5.

lower, the knockout of CaT channels would express directly as a reduction in a depolarizing current, which manifests as a reduction in excitability. A similar explanation holds for the knockout of other calcium channels as well (Fig. 6c).

Finally, with respect to both measures of coincidence detection (Fig. $6 \mathrm{~d}-\mathrm{e}$ ), $T_{\mathrm{TCDW}}$ and the $T_{\mathrm{ECDW}}$, the HCN and KA VKMs showed a large, consistent increase with respect to the corresponding baseline values. In the case of the HCN VKMs, these findings tally with prior single-sensitivity computational results from hippocampal pyramidal neurons ${ }^{23,24}$. The other VKMs exhibited small increases in $T_{\mathrm{TCDW}}$ and $T_{\mathrm{ECDW}}$.

\section{Discussion}

Our study has demonstrated (through biophysically and physiologically constrained models) that neurons possessing (1) similar encoding characteristics (in terms of the specific set of measurements from the STA kernels), (2) similar operating characteristics (in terms of whether they are coincidence detectors or integrators) and (3) similar excitability characteristics (both frequency-dependent and independent) can exist in the absence of individual or pair-wise channel homeostasis. These results suggest that hippocampal neurons have significant degrees of freedom to achieve the specific encoding and homeostasis targets associated with their physiology; in other words, degeneracy (at the molecular level) can enable cells to exhibit similar encoding and homeostasis (functional properties at the cellular level).

Implications for degeneracy in the emergence of spike-triggered average. Our study provides lines of evidence for the existence of different structural routes, involving different sets of ion channels, to achieve similar spike-triggered average and similar excitability characteristics. The many-to-many mapping between ion channels and functional measurements (Fig. 6) provide the substrate for the existence of these different solutions. The stochastic search strategy that we employed to arrive at this evidence for expression of degeneracy, however, does not address the question of how neurons organize their channel densities to arrive at these distinct solutions. It has been postulated that a general requirement for systems manifesting degeneracy is a closed-loop continual 
error-correction feedback signal that conveys whether a specific functional outcome is being achieved or not. This error signal is then employed by the system to organize its parametric space to achieve a given function, with the specific structural components chosen to achieve the function determined by the internal state of the system, neuromodulatory tones and the nature of afferent signals. A specific instance of such a broad description is with reference to the role of calcium homeostasis in maintaining channel densities through calcium-dependent transcription that is dependent on a calcium error signal ${ }^{7,8,10,15,44}$. Here, it has been shown that the solutions achieved by neurons and their networks could recruit disparate channels and receptors depending on the specific components of the network and the nature of afferent signals ${ }^{15,44}$. Although calcium constitutes one possible error correcting feedback mechanism, other possibilities including membrane voltage ${ }^{45}$ and firing rate ${ }^{46}$ could act as conduits for conveying such error signals involving their homeostasis ${ }^{47,48}$. The specific closed-loop feedback pathway could involve transcriptional networks, neuronal networks regulating excitation-inhibition balance and well-established interaction motifs widely expressed in these network structures ${ }^{49-51}$.

A neuron in particular, or a biological system in general, executes different functions in different contextual settings, with the context defined by the state of the system and the nature of external inputs. Physiological measurements, including those of excitability or operating mode, are designed to assess specific characteristics of neurons. It has been shown in several scenarios that the ability of a neuron to maintain one measurement does not necessarily translate to its ability to maintain another. For instance, there are scenarios where calcium homeostasis is maintained without homeostasis of firing rate or firing pattern, or scenarios where one model measurement is observed to match electrophysiological counterparts but others don' $\mathrm{t}^{15,44}$. This dissociation in different modes of homeostasis implies that models have to explicitly account for different measurements, and tuning of one of them doesn't necessarily maintain all measurements at physiological levels ${ }^{8,15}$. Such dissociation also implies that multi-parametric stochastic searches would, in general, yield lesser valid models as additional physiological objectives are added to the validation process (e.g., Supplementary Fig. S1).

In our model, for instance, we employed 12 intrinsic measurements (Table 2) to validate models sampled through stochastic search. In this process, owing to the dissociation of different forms of homeostasis, there were models that satisfy 11 of the measurements, but not the $12^{\text {th }}$ measurement. Does this imply that the degeneracy reported here would reduce as additional physiological constraints are included to the validation process? Degeneracy refers to the ability of different structural components to elicit similar function, and is by definition specific to the function in hand ${ }^{1,8}$. Degeneracy also implies that the same structure could be involved in the execution of a different function under a different context. Based on these definitions, it is evident that the presence of additional functional constraints on the models certainly reduces the number of valid models that satisfy all the constraints; however, this doesn't necessarily translate to reduction or abolition of degeneracy with reference to specific functions or their combinations.

The expression of degeneracy is dependent on the ability of distinct structural components to alter a specific function, implying that the presence of a many-to-one relationship between structures and a specific function is a sufficient substrate for the expression of degeneracy. In our case, we observe that several channels alter each functional measurement assessed (Fig. 6), implying the expression of degeneracy for each of these individual functional measurements. In addition, we also note that changes in each of the individual channels alter several functions in a manner that is dependent on the model parameters, suggesting a many-to-many relationship between structure and function. Furthermore, in our analyses, we have employed only a subset of parameters available for the neuron in regulating its functions. If the stochastic search space were to be increased to account for these additional parameters (other ion channels, receptors, neuromodulators, afferent input patterns, etc.), and heterogeneities therein, then the degrees of freedom available to the neuron to achieve specific functional measurements also increase in combinatorial fashion (owing to strong interactions between constituent components). This explosion in degrees of freedom then provides additional routes to fulfilling the multiple functional constraints (either in the same context or under different contexts). Finally, the cell-to-cell and animal-to-animal variability in constituent components, despite the expression of similar functional outcomes, provide lines of evidence for the expression of degeneracy in biological systems $s^{1,7,8,16,17,52}$.

Impact of individual ion channels on spike-triggered average. Our analyses show that removal of any of the 9 ion channels in any of our valid models causes the model to shift its operating mode to the integrator end of the integrator-coincidence detection continuum. This would indicate that interactions amongst the ion channels modulate the STA-based properties of a neuron. However, the HCN and KA ion channels were found to be the dominant regulators of a CA1 neuron model's operation mode, since absence of either ion channel imparts pronounced integrator-like traits, such as very low $f_{\mathrm{STA}}(\sim 1 \mathrm{~Hz})$ and broad coincidence detection windows.

The HCN channel has been shown to impart coincidence detector traits to hippocampal CA1 neurons ${ }^{23,24}$. Our results are consistent with these studies, as HCN VKMs lacked coincidence detector traits. On the other hand, an increase in KA conductance has been shown to cause an increase in the resonance frequency ${ }^{42}$, but results in a decrease in the $f_{\mathrm{STA}}{ }^{24}$. Our observations, however, suggest that eliminating the KA conductance results in a consistent reduction in $f_{\text {STA }}$, while also resulting in a broadening of the coincidence detection windows. The dependence of frequency-dependent and frequency-independent excitability measurements on different ion channels ${ }^{13-15,42}$, the role of different ion channels in regulating STA measurements ${ }^{23-25}$, and the dissociation between subthreshold resonance and STA spectral selectivity $y^{24-26}$ in hippocampal pyramidal neurons have been analyzed earlier. Although an earlier study ${ }^{24}$ predicts an increase in $f_{\text {STA }}$ with a reduction in KA channel density, the model employed there did not contain all the different ion channels expressed by hippocampal neurons and therefore do not account for the several cross-channel interactions that manifest in our analyses. In addition, as the excitability of the neuron increases with the elimination of KA channels ${ }^{42,53}$, a lower variance was required for the Gaussian white noise to elicit an average firing frequency of $1-1.5 \mathrm{~Hz}$. As $f_{\text {STA }}$ is known to be directly related 
to this variance $\mathrm{e}^{23-25}$, the observed reduction in $f_{\mathrm{STA}}$ in our analyses could also be explained by the reduction of the variance of the Gaussian white noise.

As CaT channels also mediate resonating conductances ${ }^{54}$, the CaT VKMs would have been expected to respond in a manner similar to the $\mathrm{HCN} \mathrm{VKMs}{ }^{24}$. However, our simulations indicate that the HCN conductance plays a more dominant role than the CaT conductance in mediating a CA1 pyramidal neuron's operating mode. This could partly be attributed to the absence of calcium-activated potassium channels in the earlier study ${ }^{24}$. Calcium-activated potassium channels critically interact with calcium channels in regulating excitability of hippocampal pyramidal neurons $s^{55}$, and therefore could suppress the impact of CaT channels in significantly altering neuronal operational modes ${ }^{56}$. These postulates - about the role of cross-interactions among channels in regulating neuronal STA and the impact of the variance of Gaussian white noise in the assessment of the impact of KA channels on STA properties - could be tested with electrophysiological experiments coupled with appropriate pharmacological blockers.

Although our analyses systematically showed the dependence of specific STA measurements on individual ion channels by eliminating individual channels (Fig. 6), there were no rules or mechanisms to account for such ion channel perturbations towards compensation of specific functions. In other words, our analyses were on the acute impact of ion channel removal, with no rules to govern potential changes under chronic knockout scenarios ${ }^{44}$. Future studies could incorporate specific rules involving closed-loop feedback mechanisms correcting functional errors (see above) to assess the role of different channels in compensating for specific knockouts. Such analyses would reveal the dynamical impact of ion channel knockouts, and potential heterogeneities in such impact consequent to baseline heterogeneities in ion channel conductances in specific models ${ }^{44}$.

In summary, in this study, we demonstrated the expression of degeneracy in the concomitant emergence of excitability as well as encoding measurements in hippocampal pyramidal neurons. This demonstration was constrained biophysically by the ion channel gating and kinetic properties and physiologically validated by STA and excitability measurements, all of which were obtained from hippocampal pyramidal neurons. Our results suggest that hippocampal neurons have significant degrees of freedom to achieve the specific encoding and homeostasis targets without cross-interferences in these processes ${ }^{8}$. Although our conclusions could potentially extend to other neuronal subtypes, such extrapolations should specifically account for biophysical characteristics of ion channels expressed in those neurons and the STA and excitability measurements from there as well. Future studies could assess the potential of degeneracy in concomitantly maintaining encoding as well as excitability characteristics, especially with reference to physiological and pathological perturbations.

\section{Methods}

Neuronal base model and ion channels. A single compartmental cylindrical base model was created with length $(L)$ and diameter $(d)$ both equal to $105 \mu \mathrm{m}$. The specific membrane resistance $\left(R_{\mathrm{m}}\right)$ and specific membrane capacitance $\left(C_{\mathrm{m}}\right)$ - that constitute passive properties of the model - had values of $40 \mathrm{k} \Omega . \mathrm{cm}^{2}$ and $1 \mu \mathrm{F} /$ $\mathrm{cm}^{2}$, respectively. Twelve different types of ion channels (including leak channels with conductance $g_{\text {leak }}=1 / R_{\mathrm{m}}$ ) were inserted into this structure, with default values of channel conductances listed in Table 1 . The channels employed in this study are: fast sodium $(\mathrm{NaF})$, delayed rectifier potassium (KDR), $A$-type potassium (KA), $M$-type potassium (KM), hyperpolarization-activated cyclic-nuclotide gated (HCN) nonspecific cation, $L$-type calcium (CaL), $N$-type calcium (CaN), $R$-type calcium (CaR), $T$-type calcium (CaT), big- (BK) and small-conductance (SK) calcium-activated potassium channels. The reversal potentials for $\mathrm{K}^{+}$and $\mathrm{Na}^{+}$were kept at $-90 \mathrm{mV}$ and $+55 \mathrm{mV}$, respectively, and the reversal potential for the $\mathrm{HCN}$ conductance was set to $-30 \mathrm{mV}$. The kinetics for different channels were constrained by respective electrophysiological measurements in hippocampal neurons for each of the channels present in our model: NaF, KDR and KA channels ${ }^{27,28,30}, \mathrm{HCN}$ channels ${ }^{29}$, CaR and CaL channels $^{27,57}$, CaT channels ${ }^{33}$, KM channels ${ }^{33,58}$, BK channels ${ }^{59}$, and CaN and SK channels ${ }^{60}$. The evolution of intracellular calcium as a function of calcium current through the voltage-gated calcium channels and its buffering was modeled as ${ }^{61-63}$ :

$$
\frac{d[\mathrm{Ca}]_{i}}{d t}=-\frac{10000 I_{C a}}{36 d p t F}+\frac{[C a]_{\infty}-[C a]_{i}}{\tau_{C a}}
$$

where $F$ is Faraday's constant, the default value of the calcium decay time constant $\tau_{C a}=33 \mathrm{~ms}$ (Table 1), $d p t=0.1 \mu \mathrm{m}$ is the depth of the shell, and $[\mathrm{Ca}]_{\infty}=100 \mathrm{nM}$ is the steady state value of $[\mathrm{Ca}]_{i}$.

Intrinsic properties. We employed 12 intrinsic physiological measurements to define and validate models (Fig. 1). Of these, 7 intrinsic measurements were excitability and frequency-selectivity measures that have been used to characterize hippocampal CA1 pyramidal neurons ${ }^{13-15,29,35,36,38,39,42}$. The other 5 measurements were STA-derived measurements, and were taken from their prior definitions ${ }^{23-25}$.

The 7 standard intrinsic measurements calculated were the input resistance $\left(R_{\mathrm{in}}\right.$, in $\left.\mathrm{M} \Omega\right)$, firing frequency for a pulse-current injection of amplitude $0 \mathrm{pA}$ and $250 \mathrm{pA}\left(f_{0}\right.$ and $f_{250}$ respectively; both in $\left.\mathrm{Hz}\right)$, the maximum impedance amplitude $\left(|Z|_{\max }\right.$, in $\left.M \Omega\right)$, resonance frequency $\left(f_{\mathrm{R}}\right.$, in $\left.\mathrm{Hz}\right)$, the resonance strength $\left(Q_{\mathrm{R}}\right.$, dimensionless) and total inductive phase $\left(\Phi_{\mathrm{L}}\right.$, in radian.Hz). $R_{\text {in }}$ was measured by noting the steady-state value of the membrane potential $\left(V_{\mathrm{ss}}\right)$ in response to sustained current input of $500 \mathrm{~ms}$ duration; the injected current $\left(I_{\text {inj }}\right)$ was varied from $+50 \mathrm{pA}$ to $-50 \mathrm{pA}$ in steps of $10 \mathrm{pA}$. The slope of a linear fit to the plot of $V_{\mathrm{ss}} v s . I_{\text {inj }}$ was taken to be the input resistance. Firing frequency was assessed by measuring the number of action potentials fired in a $1000 \mathrm{~ms}$ interval when a sustained pulse current was injected. In particular, firing frequency was noted for injected current values set to $0 \mathrm{pA}\left(f_{0}\right)$ and when it was $250 \mathrm{pA}\left(f_{250}\right)$. $f_{0}$ was measured to ensure that the model was not spontaneously spiking, an electrophysiological signature of CA1 pyramidal neurons ${ }^{35,38}$. To measure impedance related properties, a chirp stimulus (of constant 20-pA amplitude, but whose frequency increased linearly from $0 \mathrm{~Hz}$ to $25 \mathrm{~Hz}$ 
over a $25 \mathrm{~s}$ interval), was injected into the $\operatorname{model}^{34,35}$. The voltage response of the neuron model was recorded, and its Fourier transform was taken, along with the Fourier transform of the injected chirp current input. The Fourier transform of the voltage response was divided by that of the injected current to give the impedance. The magnitude of this impedance $Z(f)$ was computed as:

$$
|Z(f)|=\sqrt{(\operatorname{Re}(Z(f)))^{2}+(\operatorname{Im}(Z(f)))^{2}}
$$

As $Z(f)$ is a complex-valued function, $\operatorname{Re}(Z(f))$ represents the real part of this function, while $\operatorname{Im}(Z(f))$ constitutes the imaginary part. The maximum value of $|Z(f)|$ was defined as $|Z|_{\max }$. The frequency at which $|Z(f)|$ reached $|Z|_{\max }$ was called $f_{\mathrm{R}}$, the resonance frequency. The quality factor or resonance strength, $Q_{\mathrm{R}}$, was calculated to be the ratio of the maximum impedance amplitude and the impedance amplitude at $0.5 \mathrm{~Hz}^{34,35}$. The impedance phase was calculated as:

$$
\varphi(f)=\tan ^{-1} \frac{\operatorname{Im}(Z(f))}{\operatorname{Re}(Z(f))}
$$

The area under the inductive (positive) portion of the curve is termed the total inductive phase, and was computed as $^{36}$ :

$$
\Phi_{L}=\int_{\varphi(f)>0} \varphi(f) d f
$$

The STA-derived measurements were computed from prior definitions ${ }^{23-25}$ : the STA characteristic frequency $\left(f_{\mathrm{STA}}\right.$, in $\left.\mathrm{Hz}\right)$, the quality of frequency selectivity in the STA $\left(Q_{\mathrm{STA}}\right)$, the peak STA current $\left(I_{\mathrm{STA}}^{\text {peak }}\right.$, in pA), the total coincidence detection window ( $T_{\mathrm{TCDW}}$, in $\mathrm{ms}$ ) and the effective coincidence detection window ( $T_{\mathrm{ECDW}}$, in $\left.\mathrm{ms}\right)$. To determine these measurements, we first calculated the STA of each model. We did this by injecting Gaussian white noise current (Fig. 1i), whose standard deviation was adjusted to obtain a neuronal firing rate of 1-1.5 $\mathrm{Hz}^{21,23-25,64,65}$. We harvested current input from spike onset till $500 \mathrm{~ms}$ prior to it. These segments of the current input were averaged for at least 800 spikes to yield the STA. ISTA $_{\text {peak }}$ was defined as the peak STA current in the temporal representation of the STA. We took the Fourier transform of the STA, and found the characteristic frequency $\left(f_{\text {STA }}\right)$ corresponding to the peak of the Fourier magnitude. The strength of selectivity $Q_{\text {STA }}$ at this characteristic frequency was calculated as the ratio between the peak STA magnitude $\mid$ STA $\left(f_{\text {STA }}\right) \mid$ and $\mid$ STA $(0.5 \mathrm{~Hz})$. The $T_{\mathrm{TCDW}}$ was calculated as the temporal distance between the action potential (i.e., $t=0$ on the temporal representation of the STA) and the point at which the STA curve first crossed zero from the action potential. The $T_{\mathrm{ECDW}}$ was measured by weighting the $T_{\mathrm{TCDW}}$ by the amplitude of the STA within the positive region proximal to the action potential, and was computed $\mathrm{as}^{23-25}$ :

$$
T_{\mathrm{ECDW}}=\sqrt{\frac{\int_{-T_{\mathrm{TCDW}}}^{0} t^{2} \mathrm{STA}^{2}(t) d t}{\int_{-T_{\mathrm{TCDW}}}^{0} \operatorname{STA}^{2}(t) d t}}
$$

Action potential amplitude $\left(V_{\mathrm{AP}}\right.$ in $\left.\mathrm{mV}\right)$ was measured as the difference between the resting membrane potential and that at the peak of an action potential.

Multi-parametric, multi-objective stochastic search (MPMOSS). Several search strategies have been employed to assess and reveal the manifestation of degeneracy in the emergence of cellular scale physiological properties from molecular scale components ${ }^{4,6-9,11-18,40,43,66,67}$. Of these, the multi-parametric, multi-objective stochastic search (MPMOSS) technique involves random sampling to study how different combinations of molecular-scale parameters (channel conductances, channel kinetics, calcium buffering properties, etc.) derived from specific neurons could result in the emergence of signature intrinsic measurements of these neurons. If disparate sets of multiple molecular-scale parameters (for a population of neuron models) result in the robust manifestation of multiple physiological measurements (objectives) that reflect similar neuronal physiology, this model population is degenerate with respect to the measurements ${ }^{8}$. On the other hand, specific clusters of underlying parameters with strong correlations across parametric values would point to a strongly constrained system that lacks degeneracy. It is important, however, to note that in the latter scenario, declaring the absence of degeneracy is difficult because the stochastic search might not have spanned all stretches of the multi-dimensional parametric space.

The diverse set of models with randomized parameters thus generated was then subjected to validation. To do this, we sampled 14 parameters (all 12 ion channel conductances, the specific membrane capacitance and the decay time constant of calcium dynamics) from independent uniform distributions spanning a range that was typically $0.5 \times-2.0 \times$ of their respective base model values (Table 1 ). All other parameters, including the dimensions of the neuronal model, the reversal potentials and channel kinetics, were kept fixed across all randomized models. We generated 5000 unique models employing such stochastic sampling, with each of the 14 parameters randomly picked from the respective distributions. We computed all the 12 intrinsic measurements from each of these 5000 models. Finally, we determined the validity of each model by checking if all the 12 intrinsic measurements of the model fell within corresponding experimentally obtained bounds for hippocampal CA1 pyramidal neurons (Table 2). Apart from these 12 validation criteria, we imposed an additional constraint, in that the action potential amplitude $V_{\mathrm{AP}}$ had to be $>95 \mathrm{mV}$ for the model to be valid. 
Virtual knockout model (VKM) analysis. For each model, we created a virtual knockout (for a specific ion channel) by setting the conductance of that ion channel to zero. We then determined this VKM's STA, using the techniques outlined above and computed the same five STA-based measurements $-f_{\text {STA }}, Q_{\text {STA }}, I_{\text {STA }}^{\text {peak }}, T_{\text {TCDW }}$ and $T_{\mathrm{ECDW}}$. We compared these VKM-derived measurements with the corresponding measurements from the same model before the knockout. Statistics from comparative analyses involving all 72 models were then used to glean insights about the role of specific ion channels in specific STA measurements. It should be noted that these analyses assess the impact of acute knockouts, and do not incorporate rules for compensations after channel knockouts $^{44}$.

Each of the neuron models we used for these analyses possessed 11 active ion channels (excluding the leak conductance); however, VKMs of both the NaF and the KDR ion channels were not generated. This is because knocking out either $\mathrm{NaF}$ or KDR severely impaired a neuron's ability to fire action potentials, as these ion channels play critical roles in the depolarization and repolarization phases respectively of a spike. Thus, in the absence of spikes, computation of STA becomes infeasible. Consequently, VKM analyses were carried out for each of the other nine active ion channels. We thus generated a total of $9 \times 72=648 \mathrm{VKMs}$. Of these, $13 \mathrm{VKMs}$ could not maintain a $1-1.5 \mathrm{~Hz}$ firing rate for at least a $800 \mathrm{~s}$ interval (as these models entered into depolarization-induced block), while another subset of models (comprising $14 \mathrm{VKMs}$ ) generated supra-threshold oscillations even with the injection of extremely low-variance Gaussian white noise. Both these groups of models were excluded from our analyses.

Simulation details. All simulations were performed in the NEURON simulation environment ${ }^{68}$ with the resting membrane potential set at $-65 \mathrm{mV}$, and the temperature kept at $34^{\circ} \mathrm{C}$. All simulations were run with a step size of $25 \mu \mathrm{s}$. Data analysis was carried out using Igor Pro (WaveMetrics, Inc) and statistical analyses were performed using the R statistical package ${ }^{69}$.

\section{Data availability}

All data generated or analyzed during this study are included in this published article and its Supplementary Information files. The codes employed in this study are available from the corresponding author on request.

Received: 13 May 2019; Accepted: 26 December 2019;

Published online: 15 January 2020

\section{References}

1. Edelman, G. M. \& Gally, J. A. Degeneracy and complexity in biological systems. Proc. Natl Acad. Sci. USA 98, 13763-13768, https:// doi.org/10.1073/pnas.231499798 (2001).

2. Price, C. J. \& Friston, K. J. Degeneracy and cognitive anatomy. Trends Cogn. Sci. 6, 416-421 (2002).

3. Leonardo, A. Degenerate coding in neural systems. J. Comp. Physiol. A, Neuroethology, sensory, neural, Behav. physiology 191, 995-1010, https://doi.org/10.1007/s00359-005-0026-0 (2005).

4. Taylor, A. L., Goaillard, J. M. \& Marder, E. How multiple conductances determine electrophysiological properties in a multicompartment model. J. Neurosci. 29, 5573-5586, https://doi.org/10.1523/JNEUROSCI.4438-08.2009 (2009).

5. Whitacre, J. \& Bender, A. Degeneracy: a design principle for achieving robustness and evolvability. J. Theor. Biol. 263, 143-153, https://doi.org/10.1016/j.jtbi.2009.11.008 (2010).

6. Drion, G., O'Leary, T. \& Marder, E. Ion channel degeneracy enables robust and tunable neuronal firing rates. Proc. Natl Acad. Sci. USA 112, E5361-5370, https://doi.org/10.1073/pnas.1516400112 (2015).

7. O'Leary, T. Homeostasis, failure of homeostasis and degenerate ion channel regulation. Curr. Opin. Physiol. 2, 129-138 (2018).

8. Rathour, R. K. \& Narayanan, R. Degeneracy in hippocampal physiology and plasticity. Hippocampus 29, 980-1022, https://doi. org/10.1101/203943 (2019).

9. Mukunda, C. L. \& Narayanan, R. Degeneracy in the regulation of short-term plasticity and synaptic filtering by presynaptic mechanisms. J. Physiol. 595, 2611-2637, https://doi.org/10.1113/JP273482 (2017)

10. O'Leary, T., Williams, A. H., Caplan, J. S. \& Marder, E. Correlations in ion channel expression emerge from homeostatic tuning rules. Proc. Natl Acad. Sci. USA 110, E2645-2654, https://doi.org/10.1073/pnas.1309966110 (2013).

11. Foster, W. R., Ungar, L. H. \& Schwaber, J. S. Significance of conductances in Hodgkin-Huxley models. J. Neurophysiol. 70, 2502-2518 (1993).

12. Goldman, M. S., Golowasch, J., Marder, E. \& Abbott, L. F. Global structure, robustness, and modulation of neuronal models. J. Neurosci. 21, 5229-5238, https://doi.org/10.1523/JNEUROSCI.21-14-05229.2001 (2001).

13. Rathour, R. K. \& Narayanan, R. Inactivating ion channels augment robustness of subthreshold intrinsic response dynamics to parametric variability in hippocampal model neurons. J. Physiol. 590, 5629-5652, https://doi.org/10.1113/jphysiol.2012.239418 (2012).

14. Rathour, R. K. \& Narayanan, R. Homeostasis of functional maps in active dendrites emerges in the absence of individual channelostasis. Proc. Natl Acad. Sci. USA 111, E1787-1796, https://doi.org/10.1073/pnas.1316599111 (2014).

15. Srikanth, S. \& Narayanan, R. Variability in State-Dependent Plasticity of Intrinsic Properties during Cell-Autonomous SelfRegulation of Calcium Homeostasis in Hippocampal Model Neurons. eNeuro 2, ENEURO.0053-0015.2015, https://doi.org/10.1523/ ENEURO.0053-15.2015 (2015)

16. Marder, E. Variability, compensation, and modulation in neurons and circuits. Proc. Natl Acad. Sci. USA 108(Suppl 3), 15542-15548, https://doi.org/10.1073/pnas.1010674108 (2011)

17. Marder, E. \& Goaillard, J. M. Variability, compensation and homeostasis in neuron and network function. Nat. reviews. Neurosci. 7, 563-574 (2006).

18. Prinz, A. A., Bucher, D. \& Marder, E. Similar network activity from disparate circuit parameters. Nat. Neurosci. 7, 1345-1352, https://doi.org/10.1038/nn1352 (2004).

19. Migliore, R. et al. The physiological variability of channel density in hippocampal CA1 pyramidal cells and interneurons explored using a unified data-driven modeling workflow. PLoS computational Biol. 14, e1006423, https://doi.org/10.1371/journal. pcbi.1006423 (2018).

20. Ratte, S., Hong, S., De Schutter, E. \& Prescott, S. A. Impact of neuronal properties on network coding: roles of spike initiation dynamics and robust synchrony transfer. Neuron 78, 758-772, https://doi.org/10.1016/j.neuron.2013.05.030 (2013).

21. Hong, S. Aguera y Arcas, B. \& Fairhall, A. L. Single neuron computation: from dynamical system to feature detector. Neural Comput. 19, 3133-3172, https://doi.org/10.1162/neco.2007.19.12.3133 (2007). 
22. Hong, S., Ratte, S., Prescott, S. A. \& De Schutter, E. Single neuron firing properties impact correlation-based population coding. J. Neurosci. 32, 1413-1428, https://doi.org/10.1523/JNEUROSCI.3735-11.2012 (2012).

23. Das, A. \& Narayanan, R. Active dendrites regulate spectral selectivity in location-dependent spike initiation dynamics of hippocampal model neurons. J. Neurosci. 34, 1195-1211, https://doi.org/10.1523/JNEUROSCI.3203-13.2014 (2014).

24. Das, A. \& Narayanan, R. Active dendrites mediate stratified gamma-range coincidence detection in hippocampal model neurons. J. Physiol. 593, 3549-3576, https://doi.org/10.1113/JP270688 (2015).

25. Das, A. \& Narayanan, R. Theta-frequency selectivity in the somatic spike triggered average of rat hippocampal pyramidal neurons is dependent on HCN channels. J. Neurophysiol. 118, 2251-2266, https://doi.org/10.1152/jn.00356.2017 (2017).

26. Das, A., Rathour, R. K. \& Narayanan, R. Strings on a Violin: Location Dependence of Frequency Tuning in Active Dendrites. Front. Cell Neurosci. 11, 72, https://doi.org/10.3389/fncel.2017.00072 (2017).

27. Magee, J. C. \& Johnston, D. Characterization of single voltage-gated $\mathrm{Na}+$ and $\mathrm{Ca} 2+$ channels in apical dendrites of rat CA1 pyramidal neurons. J. Physiol. 487(Pt 1), 67-90 (1995).

28. Hoffman, D. A., Magee, J. C., Colbert, C. M. \& Johnston, D. K+ channel regulation of signal propagation in dendrites of hippocampal pyramidal neurons. Nature 387, 869-875 (1997).

29. Magee, J. C. Dendritic hyperpolarization-activated currents modify the integrative properties of hippocampal CA1 pyramidal neurons. J. Neurosci. 18, 7613-7624 (1998).

30. Migliore, M., Hoffman, D. A., Magee, J. C. \& Johnston, D. Role of an A-type K+ conductance in the back-propagation of action potentials in the dendrites of hippocampal pyramidal neurons. J. Comput. Neurosci. 7, 5-15 (1999).

31. Migliore, M. \& Shepherd, G. M. Emerging rules for the distributions of active dendritic conductances. Nat. reviews. Neurosci. 3 , $362-370$ (2002)

32. Migliore, M. \& Shepherd, G. M. Opinion: an integrated approach to classifying neuronal phenotypes. Nat. reviews. Neurosci. 6 , 810-818 (2005)

33. Shah, M. M., Migliore, M. \& Brown, D. A. Differential effects of Kv7 (M-) channels on synaptic integration in distinct subcellular compartments of rat hippocampal pyramidal neurons. J. Physiol. 589, 6029-6038, https://doi.org/10.1113/jphysiol.2011.220913 (2011).

34. Hu, H., Vervaeke, K. \& Storm, J. F. Two forms of electrical resonance at theta frequencies, generated by M-current, h-current and persistent $\mathrm{Na}+$ current in rat hippocampal pyramidal cells. J. Physiol. 545, 783-805 (2002).

35. Narayanan, R. \& Johnston, D. Long-term potentiation in rat hippocampal neurons is accompanied by spatially widespread changes in intrinsic oscillatory dynamics and excitability. Neuron 56, 1061-1075 (2007).

36. Narayanan, R. \& Johnston, D. The h channel mediates location dependence and plasticity of intrinsic phase response in rat hippocampal neurons. J. Neurosci. 28, 5846-5860 (2008).

37. Narayanan, R. \& Johnston, D. Functional maps within a single neuron. J. Neurophysiol. 108, 2343-2351, https://doi.org/10.1152/ jn.00530.2012 (2012)

38. Narayanan, R., Dougherty, K. J. \& Johnston, D. Calcium Store Depletion Induces Persistent Perisomatic Increases in the Functional Density of h Channels in Hippocampal Pyramidal Neurons. Neuron 68, 921-935, https://doi.org/10.1016/j.neuron.2010.11.033 (2010).

39. Malik, R., Dougherty, K. A., Parikh, K., Byrne, C. \& Johnston, D. Mapping the electrophysiological and morphological properties of CA1 pyramidal neurons along the longitudinal hippocampal axis. Hippocampus 26, 341-361, https://doi.org/10.1002/hipo.22526 (2016).

40. Anirudhan, A. \& Narayanan, R. Analogous synaptic plasticity profiles emerge from disparate channel combinations. J. Neurosci. 35, 4691-4705, https://doi.org/10.1523/JNEUROSCI.4223-14.2015 (2015).

41. Evans, J. D. Straightforward statistics for the behavioral sciences. (Brooks/Cole Pub Co, 1996).

42. Rathour, R. K., Malik, R. \& Narayanan, R. Transient potassium channels augment degeneracy in hippocampal active dendritic spectral tuning. Sci. Rep. 6, 24678, https://doi.org/10.1038/srep24678 (2016).

43. Basak, R. \& Narayanan, R. Spatially dispersed synapses yield sharply-tuned place cell responses through dendritic spike initiation. J. Physiol. 596, 4173-4205, https://doi.org/10.1113/JP275310 (2018).

44. O’Leary, T., Williams, A. H., Franci, A. \& Marder, E. Cell types, network homeostasis, and pathological compensation from a biologically plausible ion channel expression model. Neuron 82, 809-821, https://doi.org/10.1016/j.neuron.2014.04.002 (2014).

45. Santin, J. M. \& Schulz, D. J. Membrane Voltage Is a Direct Feedback Signal That Influences Correlated Ion Channel Expression in Neurons. Curr. biology: CB 29, 1683-1688 e1682, https://doi.org/10.1016/j.cub.2019.04.008 (2019).

46. Hengen, K. B., Torrado Pacheco, A., McGregor, J. N., Van Hooser, S. D. \& Turrigiano, G. G. Neuronal Firing Rate Homeostasis Is Inhibited by Sleep and Promoted by Wake. Cell 165, 180-191, https://doi.org/10.1016/j.cell.2016.01.046 (2016).

47. Gjorgjieva, J., Drion, G. \& Marder, E. Computational implications of biophysical diversity and multiple timescales in neurons and synapses for circuit performance. Curr. Opin. Neurobiol. 37, 44-52, https://doi.org/10.1016/j.conb.2015.12.008 (2016).

48. Turrigiano, G. Too many cooks? Intrinsic and synaptic homeostatic mechanisms in cortical circuit refinement. Annu. Rev. Neurosci. 34, 89-103, https://doi.org/10.1146/annurev-neuro-060909-153238 (2011).

49. Milo, R. et al. Network motifs: simple building blocks of complex networks. Science 298, 824-827, https://doi.org/10.1126/ science.298.5594.824 (2002)

50. Ma'ayan, A. et al. Formation of regulatory patterns during signal propagation in a Mammalian cellular network. Science 309, 1078-1083, https://doi.org/10.1126/science.1108876 (2005).

51. Bullmore, E. \& Sporns, O. Complex brain networks: graph theoretical analysis of structural and functional systems. Nat. reviews. Neurosci. 10, 186-198, https://doi.org/10.1038/nrn2575 (2009).

52. Marder, E. \& Taylor, A. L. Multiple models to capture the variability in biological neurons and networks. Nat. Neurosci. 14, 133-138, https://doi.org/10.1038/nn.2735 (2011).

53. Chen, X. et al. Deletion of Kv4.2 gene eliminates dendritic A-type K+ current and enhances induction of long-term potentiation in hippocampal CA1 pyramidal neurons. J. Neurosci. 26, 12143-12151 (2006).

54. Hutcheon, B. \& Yarom, Y. Resonance, oscillation and the intrinsic frequency preferences of neurons. Trends Neurosci. 23, 216-222 (2000).

55. Adelman, J. P., Maylie, J. \& Sah, P. Small-conductance Ca2+-activated K+ channels: form and function. Annu. Rev. Physiol. 74, 245-269, https://doi.org/10.1146/annurev-physiol-020911-153336 (2012).

56. Prescott, S. A. \& Sejnowski, T. J. Spike-rate coding and spike-time coding are affected oppositely by different adaptation mechanisms. J. Neurosci. 28, 13649-13661, https://doi.org/10.1523/JNEUROSCI.1792-08.2008 (2008).

57. Poirazi, P., Brannon, T. \& Mel, B. W. Pyramidal neuron as two-layer neural network. Neuron 37, 989-999 (2003).

58. Migliore, M., Cannia, C., Lytton, W. W., Markram, H. \& Hines, M. L. Parallel network simulations with NEURON. J. Comput. Neurosci. 21, 119-129, https://doi.org/10.1007/s10827-006-7949-5 (2006)

59. Moczydlowski, E. \& Latorre, R. Gating kinetics of Ca2+-activated $\mathrm{K}+$ channels from rat muscle incorporated into planar lipid bilayers. Evidence for two voltage-dependent Ca2+ binding reactions. J. Gen. Physiol. 82, 511-542 (1983).

60. Migliore, M., Cook, E. P., Jaffe, D. B., Turner, D. A. \& Johnston, D. Computer simulations of morphologically reconstructed CA3 hippocampal neurons. J. Neurophysiol. 73, 1157-1168 (1995)

61. Poirazi, P., Brannon, T. \& Mel, B. W. Arithmetic of subthreshold synaptic summation in a model CA1 pyramidal cell. Neuron 37, 977-987 (2003). 
62. Narayanan, R. \& Johnston, D. The h current is a candidate mechanism for regulating the sliding modification threshold in a BCMlike synaptic learning rule. J. Neurophysiol. 104, 1020-1033, https://doi.org/10.1152/jn.01129.2009 (2010).

63. Honnuraiah, S. \& Narayanan, R. A calcium-dependent plasticity rule for $\mathrm{HCN}$ channels maintains activity homeostasis and stable synaptic learning. PLoS one 8, e55590, https://doi.org/10.1371/journal.pone.0055590 (2013).

64. Aguera y Arcas, B. \& Fairhall, A. L. What causes a neuron to spike? Neural Comput. 15, 1789-1807, https://doi. org $/ 10.1162 / 08997660360675044$ (2003).

65. Famulare, M. \& Fairhall, A. Feature selection in simple neurons: how coding depends on spiking dynamics. Neural Comput. 22, 581-598, https://doi.org/10.1162/neco.2009.02-09-956 (2010).

66. Mittal, D. \& Narayanan, R. Degeneracy in the robust expression of spectral selectivity, subthreshold oscillations and intrinsic excitability of entorhinal stellate cells. J. Neurophysiol. 120, 576-600, https://doi.org/10.1152/jn.00136.2018 (2018).

67. Mishra, P. \& Narayanan, R. Disparate forms of heterogeneities and interactions among them drive channel decorrelation in the dentate gyrus: Degeneracy and dominance. Hippocampus 29, 378-403, https://doi.org/10.1002/hipo.23035 (2019).

68. Carnevale, N. T. \& Hines, M. L. The NEURON Book. (Cambridge University Press, 2006).

69. R Core Team. R: A Language and Environment for Statistical Computing. (2014).

\section{Acknowledgements}

This work was supported by the Wellcome Trust-DBT India Alliance (Senior fellowship to RN; IA/S/16/2/502727), Human Frontier Science Program (HFSP) Organization (RN), the Department of Biotechnology through the DBT-IISc partnership program (RN), the Department of Science and Technology (RN), the Ministry of Human Resource Development (RN) and Kishore Vaigyanik Protsahan Yojana (AJ).

\section{Author contributions}

A.J. and R.N. designed experiments; A.J. performed experiments and carried out data analysis; A.J. and R.N. cowrote the paper.

\section{Competing interests}

The authors declare no competing interests.

\section{Additional information}

Supplementary information is available for this paper at https://doi.org/10.1038/s41598-019-57243-8.

Correspondence and requests for materials should be addressed to R.N.

Reprints and permissions information is available at www.nature.com/reprints.

Publisher's note Springer Nature remains neutral with regard to jurisdictional claims in published maps and institutional affiliations.

Open Access This article is licensed under a Creative Commons Attribution 4.0 International License, which permits use, sharing, adaptation, distribution and reproduction in any medium or format, as long as you give appropriate credit to the original author(s) and the source, provide a link to the Creative Commons license, and indicate if changes were made. The images or other third party material in this article are included in the article's Creative Commons license, unless indicated otherwise in a credit line to the material. If material is not included in the article's Creative Commons license and your intended use is not permitted by statutory regulation or exceeds the permitted use, you will need to obtain permission directly from the copyright holder. To view a copy of this license, visit http://creativecommons.org/licenses/by/4.0/.

(C) The Author(s) 2020 